\section{Nachhaltige Entwicklung durch endlose Produktkreisläufe in der Technosphäre?}

\author{
M. Braungart, W. McDonough: Intelligen- \\ te Verschwendung. The Upcycle: Auf \\ dem Weg in eine neue Überflussgesell- \\ schaft. München: oekom verlag 2013, 208 \\ S., ISBN 978-3-8658-1316-9, Euro 17,95
}

\section{Rezension von Jens Buchgeister, ITAS}

\section{Zielsetzung}

Die Autoren des im Folgenden rezensierten $\mathrm{Bu}-$ ches verstehen unter dem Begriff Upcycling

„eine wunderbar vielfältige, sichere, gesunde und gerechte Welt mit sauberer Luft, sauberem Wasser, sauberem Boden und sauberer Energie eine Welt derer wir uns in vielfacher Weise und in grenzenloser Harmonie erfreuen können“" (S. 9).

Dies darf als eine Vision im Sinne einer vollständigen Umsetzung und Implementierung einer nachhaltigen Entwicklung für sämtliche Lebensbedürfnisse auch zukünftiger Generationen weltweit verstanden werden. Auf dem Weg zur Umsetzung der Vision setzen die Autoren weder auf eine radikale Verhaltensänderung der Menschen unter Anwendung einer Suffizienzstrategie noch auf einen radikalen Wechsel des bestehenden kapitalistischen Wirtschaftssystems mit ihrer industriellen Produktion. Ganz im Gegenteil, denn es seien „Produkte zu entwickeln, die zum Überfluss in der Welt beitragen“. Die Autoren sehen das Heil darin, den Blick immer auch aus systemischer Perspektive auf die Gestaltung jeglicher Art von Produkten, Infrastrukturen und Dienstleistungen durch Konstrukteure, Entwickler, Architekten, Planer, Designer oder ganz allgemein den Erfinder zu richten.

„Es steht fest, dass wir zu einem Design imstande sind, das besser für den Planeten ist. Wir wissen, es steht in unserer Macht, es zu tun.“(S. 148)

So wie es im Laufe einer langen Evolutionszeit gelungen sei, alle lebenswichtigen stofflichen Prozesse in Zyklen des biologischen Kreislaufs umzusetzen, solle der Mensch die geschaffene Technosphäre in einen ,technischen Metabolismus“ überführen. Das heißt, ein solcher Metabolismus kennt keinen stofflichen Abfall mehr. Alle hergestellten Produkte beinhalten keine toxischen Substanzen und lassen sich ohne Gefahren quasi als „Technische Nährstoffe“ kompostieren oder „können endlos“ auf gleichem oder höherem Niveau ,wiederverwertet werden“. Dies bedeute für ein Unternehmen, beispielsweise eine Textilfabrik, dass das eingesetzte Wasser im Unternehmen, dieses ,genauso sauber oder sauberer als das Wasser, das man hineinleitet, verlässt".

\section{These}

Die These ist, dass die menschliche Gestaltungskreativität so groß ist, dass sich im Sinne einer auf Werte beruhenden Nachhaltigkeit ein „Upcycling" in den bestehenden Wirtschaftsstrukturen bei jeglicher Art von Produkten erreichen lässt.

„Auf Werten basiertes Design nützt allen Menschen und Spezies (oder wird ihnen während einer Übergangsphase wenigstens nicht schaden). ...Würde man die industrielle Produktion als etwas Negatives betrachten, wenn ihre Ergebnisse nützlich und harmlos wären? Natürlich nicht. Eine Waschmaschine bringt einen echten Nutzen. Dasselbe gilt für den Computer, das Auto und viele andere Dinge, die wir schätzen. Der Wunsch, diese Dinge zu besitzen, ist nicht dekadent, sondern ein völlig legitimes Verlangen nach mehr Bequemlichkeit und Lebensgenuss. Ein Überfluss - an Menschen, an Produkten ist kein Übel: Die Gesellschaft kann sogar Hunderttausende Produkte aus Tausenden Kulturen aufnehmen und fördern und jedem der 10 Milliarden Menschen gerecht werden, die noch in diesem Jahrhundert die Erde bevölkern werden. Tatsächlich wird ihre Zahl durch unseren physischen und kreativen Ideenreichtum bestimmt, durch die Dinge um uns herum und unsere Fähigkeit, sinnvoll und produktiv mit ihnen umzugehen. Eine Cradle to Cradle-Welt würde es den Menschen ermöglichen, den Überfluss zu genießen und Vielfalt zu fördern, ohne dass die Welt dabei verarmt.“ (S. 141)

\section{Methodologie}

Für das auf Basis von Werten auszurichtende und durchdachte Design wird auf den Cradle to Cradle-Ansatz verwiesen, der ausführlich in dem, von den Autoren zuerst veröffentlichten Buch behandelt wird, und eine Ideensammlung für die Produktgestaltung beinhaltet. ${ }^{1}$ 
Die Autoren verfolgen mit dem Cradle to Cradle-Ansatz zwei Anliegen:

1. Ihnen ist es wichtig, den Gestaltungsansatz „weniger schlecht" durch einen Positivansatz, wie die Produkte ,mehr gut" sein können, zu ersetzen. Hierbei messen die Autoren den eingesetzten Materialien eine besonders große Bedeutung bei, da in vielen Fällen kein hochwertiges Recyceln, sondern nur ein Downcycling der Materialien möglich sei. Deshalb schlagen die Autoren eine Positivliste von einzusetzenden Materialien vor, um eine Verunreinigung auf elementarer oder molekularer Weise von Materialien in höchstem Maße in der Technosphäre zu vermeiden.

2. Der Gestaltungsprozess ist auf allen Ebenen und für alle Produkte anzuwenden. Hierbei sollen sich die Unternehmen von einengenden Gedankenmustern, z. B. dem kurzfristigen Erfolg lösen und einen alternativen Plan verfolgen, in dem „Prinzipien, Ziele, Strategien und Taktik im Anschluss einer vorangestellten Diskussion um Werte, für die sich das Unternehmen engagieren will“, formuliert werden.

In diesen beiden Grundsätzen liegt die größte Unterscheidung zu dem, in der Technikfolgenabschätzung bekannten, systemanalytischen Instrument der Lebensweganalyse bzw. Life Cycle Assessment (DIN ISO 14040 und 14044), die das Produkt von der Wiege bis zur Bahre (Cradle to Grave) über den gesamten Lebensweg hinsichtlich seiner Umweltauswirkungen bewertet. Dieses Instrument dient zur Analyse des Ist-Stands eines Produktlebenswegs und verfolgt das Ziel, die größten Einzelbeiträge, z. B. der Lebenswegphasen oder von einzelnen Prozessen an den Gesamtumweltauswirkungen, aufzudecken. Hierbei werden die Ergebnisse auf die vorher zu definierende funktionelle Einheit des Produkts bezogen. Die Lebensweganalyse trifft keine Aussage, welche Gesamtmengen an Umweltauswirkungen für den Planet tragfähig sind, da es ein relativer Ansatz ist. Erst nach der Analyse und Auswertung der Ergebnisse unterstützt das Instrument, an welchen Stellen die Verbesserung der Produktgestaltung ansetzen kann. Bei diesem Prozess lässt sich die, von den Autoren gewünschte Vorgehensweise unter zweitens durchführen. Ebenso ließen sich mittels Life
Cycle Assessment die neuentwickelten Produkte bewerten und mit den Vorgängern vergleichen.

\section{Fazit}

Anhand einer Vielzahl von unterschiedlichen Produktbeispielen erläutern die Autoren, wie aus ihrer Sicht der Cradle to Cradle-Ansatz zu einer neuen Strategie der Produktgestaltung führt und in welcher Weise der Begriff des „Upcyclings“ bei den spezifischen Produkten umzusetzen sei. Hierbei wird bei einigen Fallbeispielen, z. B. für die Ausgestaltung der Stromversorgung oder des Werkstoffs Aluminium die Detailtiefe vermisst, die nötig wäre, um zu erkennen, in welcher Weise eine Umsetzung des Ansatzes erfolgen könnte.

Außerdem wird eine theoretische Abhandlung und kritische Auseinandersetzung vermisst, ob sich der visionäre Produktgestaltungsansatz des „Upcyclings" auf alle in der Technosphäre befindlichen Produkte anwenden lässt und ob es dazu keine Veränderungen der politischen und gesellschaftlichen Rahmenbedingungen des bestehenden Wirtschaftssystems bedürfte, wie in vielen Beiträgen der Nachhaltigkeitsdiskussion zu lesen ist.

Was für den Leser so gar nicht nachvollziehbar ist, ist auf den Seiten 84 bis 88 die ausführliche Erklärung des Autoreninteresses „Überfluss auf allen Ebenen zu schaffen" und das damit intendierte Geschäftsmodell der Autoren. Dies führt konsequenterweise zur Firmengründung der McDonough Braungart Chemistry Design (MBCD), um Organisationen, insbesondere Unternehmen bei der Umsetzung einer Cradle to Cradle-Strategie innerhalb des spezifischen Prozesses der Produkt(neu)gestaltung zu unterstützen. Die Dienstleistung hat zum Ziel, am Ende des Prozesses ein Produktzertifikat zu erreichen, das öffentlich gemacht werden soll.

\section{Anmerkung}

1) Das hier rezensierte Buch ist die Fortsetzung des ersten von den Autoren Michael Braungart und William McDonough erschienenen Buches „Cradle to Cradle. Einfach intelligent produzieren“ aus dem Jahre 2002. 